\title{
VIII. Ueber den Verlauf der Choleraforschung seit der Koch'schen Expedition und Ent- deckung des Kommabacillus bis zum Schlusse des Jahres 1885.
}

Von

Dr. A. Pfeiffer-Wiesbaden.

(Fortsetzung aus No. 9.)

Als Repräsentanten der von mir aufgestellten zweiten Kategorie der Gegner der Koch'schen Entdeckung, deren Behauptung ich oben dahin präcisirt hatte: dass der Koch'sche Kommabacillus absolut Nichts mit der Aetiologie der Cholera indica zu thimn labe, oder wenigstens nur als ein gleichzeitiger aber durchaus nebensächlicher Befund zu betrachteı sei, treten hauptsächlich zwei Arbeiten in den Vordergrund. In der ersten derselben hat Herr J. M. Cuningham, Generalarzt und Medicinalreferent bei der indischen Regierung seine Ansichten niedergelegt. Es genügt, ans dem, mit einem Vorwort Pettenk ofer's verseheneı Werkchen folgenden Passus zu citirell. Cuningluam sagt auf Seite 110 des Buches "Die Cholera, was kanll der Staat thun sie zu verhïten " wörtlich:

„In dem bisher Gesagten wurden Koch's Aufstellungen, insofern sie sich an Thatsachen hieltell, acceptirt, als ob sie ïber alle Zweifel klar bewiesen wären. Aber seit diese Paragraphen geschrieben wurden, sind die stärksten Beweise gegell ihre Riclitigkeit voll verschiedenen Seiten erbracht worden. Dr. Lewis, dessen Arbeiten in Bezug auf die Cholera schon Erwähnung geschah, hat gezeigt, dass ein Spirillum, das in allen seinen mikroskopischen Eigenschaften von Koch's Cholerabacillns nicht unterscheidbar ist, in dem Speichel gesunder Menschen gefunden wird, und die englische ans den Herren Klein und Heneage Gibbes bestehende Choleracommission hat als Ergebniss ihrer Untersuchungen sowohl in Bombay wie in Calcutta, wo sie auf demselben Boden wie $\mathrm{Koch}$ stand, gezeigt, dass sein Bacillns auch in auderen als Cholerafällen vorkoınmt. Ferner sind Kommabacillen in dem gleichen Tank gefunden worden, in dem $\mathrm{Koch}$ sie fand, und doch ist seit vielen Monaten kein Fall von Cholera unter den Anwohnern, die ihn bellutzen, vorgekommen. Das ganze Hochgebäude, das die deutsche Choleracommission auf die Annahme errichtet, dass der Kommabacillus ein der Cholera eigenthümlicher Organismus sei und das mit so bereitwilliger Zustimmung, sowohl von dem Publikum, wie auch einem grossen Theil der Fachleute angestaunt wurde, ist thatsächlich zusammengestürzt."

Die zweite hierher gehörige Arbeit ist aus dem Münchener hygienischen Institut hervorgegangen und betrifft den seinerzeit von Seiten der Pettenk ofer'schen Schule mit grossem Enthusiasmus begriissten Neapeler Cholerabacillus von $\mathrm{Emmerich}$, welcher bestimmt ist, die Lehre von dem Koch'schen Kommabacillus gänzlich über den Haufen zu werfen. Es ist hier nicht möglich, eine eingelende Kritik dieser Arbeit, die zudem nicht abgeschlossen ist, zu geben. Im Allgemeinen lässt sich über den bis jetzt erschienenen Theil derselben sagen, dass er nicht nur voll Seiten competenter Kritiker die gebührende Abfertignng gefunden hat, sondern auch in sich selbst so 
sehr den Charakter einer Tendenzarbeit trägt, dass es zur Kennzeichnung genügt, nachstehende, aus vielen ähnlichen herausgegriffene Behauptung wiederzngeben. Wir wissen, dass Koclı immer betont hat, dass seine ganze Beweisfülıung bezüglich des ätiologischen Verhältnisses seines Bacillus zur Cholera indica sofort zusammenfalle, sowie nur ein einziges Mal mit Sicherheit der Koclı'sche Kommabacillus ausser bei Cholera anfgefunden sei. Dem gegeniiber behauptet nun Emmerich, mit der an ihm gewöhnten Un - felllbarkeit, ${ }^{1}$ ) dass die Koch'schen Vibrionen auch im normalen Darm in beschränkter $Z$ ahl vorkämen, sich nur, begünstigt durch die, in Folge der Einwanderung der Neapeler Bacillen in den Darm angeregte, lebhafte Transsudation einer stark alkalischen, sanerstoffreichen Flüssigkeit, vermehrten und so in manchen Fällen den Auschein erweckten, als seien sie die Ursache der vorhandenen Veränderung. Von dem bekannten Entdecker der Pueumoniekokken im Füllmaterial der Fussböden, von dem Entdecker der Diphtheriebakterien, der in seinem Aufsatz ïber die Ursache der Diphtherie des Menschen und der Tauben die exacte Arbeit Löffler's über denselben Gegenstand mit den Worten abfertigt: „Die Angaben Löffler's, welcher im Kaiserlichen Gesundheits-Amt bei der Diphtherie des Menschen etc. vier verschiedene Mikroorganismen gefunden liaben wollte, mussten von vornlıerein Bedenken erregen ", der aber in demselben Aufsatz weiter sagt: „Meine Befunde dagegen, welche die Identität der Diphtheritis des Menschen und der Thiere, oder doch wenigstens der Tauben, sicher gestellt haben, finden sich im Einklang mit den klinischen und epidemiologischen Beobachtungen und mit jenen der vergleichenden patlologischen Anatomie, sie harmoniren mit den Thatsachen, welche über die specifischen Pilze derjenigen Infectionskrankheiten festgestellt wurden, welche, wie z. B. Milzbrand und Tuberculose, sowohl beim Menschen, als anch bei den Thieren vorkommen", ich sage, von diesem unfehlbaren Entdecker muss man selbst eine solch unbegreifliche Behauptung, dass der Koch'sche Kommabacillus auch im normalen Darm vorkomme, mit Ruhe himnehmen, da dieselbe den wohlfundamentirten Forschungsergebnissen $\mathrm{K}_{0} \mathrm{ch}$ 's doch ernstlich nicht gegenüber gestellt werden darf. Was den Neapeler Cholerabacillus selbst betrifft, so älmelt er, nach den Arbeiten Buchne $\left.\mathbf{r}^{\prime}{ }^{2}\right)$, in allen seinen biologischen Eigenschaften, mit Ansnahme seines Verhaltens gegen stark saure Nährflüssigkeiten, so sehr einem im menschlichen Darmkanal hänfger vorkommenden Bacillus (Bacillus G. Buchner), dass es zu deren endgültiger Unterscheidung noch eingehenderer Arbeiten, als sie bis jetzt vorliegen, bedarf. Ich kann wenigstens die Bereclitignng, aus den zwei angegebenen Versuchen (1. c. S. 431) den ,sicheren Beweis" zu entnehmen, ,dass wir es beim Neapeler Bacillus und dem Darmbacillus G. mit zwei verschiedenen Organismen zu thun haben," nicht anerkennen, zumal die Versuchsbedingungen, meiner Ansicht nach, etwas zu gewaltthätig inscenirt erscheinen.

Lasse ich nunmehr Alles, was von gegnerischer Seite behufs Einschränkung oder gänzlicher Ableugnung der weittragenden Bedentung der Koch'schen Entdeckung des Kommabacillus, sowohl fïr Aetiologie als Prophylaxe der Cholera indica, vorgebracht wurde, noch einmal im Geiste Revue passiren, so muss ich gestehen, dass selten eine wissenschaftliche Entdeckung nichtssagendere Angriffe zu erleiden geliabt hat, als die Kocli's über die Ursache der Cholera; und offen gesagt, es beschleicht mich ein gewisses Gefülll der Beschämung, dass wir diese Angriffe leider nicht nur im Auslande zn suchen haben. Für jede wissenschaftliche Entdeckung ist eine gesmude Discussion von Vortheil, und ich bin der letzte der zu blindem Autoritätenglauben neigt, aber sine ira et studio müssen die Einwände vorgebracht werden, und, was man billig verlangen kann, vorher wohl überlegt und sicher fundirt sein.

\section{(Fortsetzung folgt.)}

\section{C. Binz. Vorlesungen über Pharmakologie für} Aerzte und Studirende. Berlin, Hirscliwald, 1886. Ref. Prof. Rossbach.

Soeben liat von genanntem Werke die dritte (Schluss-) Abtheilung die Presse verlassen; und ist es nunmelr möglicl, einen Ueberblick über dasselbe zn geben. Es unterliegt keinem Zweifel, dass dasselbe einem schon längst gefiihlten Bedürfniss der ärztlichen Welt entgegenkommt und demselben in geradezn vorzüglicher Weise gerecht wird.

Alle Lehrbücher der Arzneimittellehre ohne Ansuahme (nnd Referent schliesst auch sein eigenes mit Nothnagel herausgegebenes

1) Emmerich: Untersuchungen über die Pilze der Cholera asiatica. Archiv für Hygiene, III Bd., 3. u. 4 Heft S. 358

2) Buchner: Beiträge zur Kenntniss der Neapeler Cholerabacilleu, Archiv für Hygiene, III. Bd., 3. u. 4. Heft S. 361 ff. nicht aus) sind doch eigentlich nur Nachschlagebücher, bestimmt, dem Arzte in allen möglichen nud denkbaren Fällen Anfschluss über die Wirkung der angewendeten Mittel nach allen möglichen Richtungen, auf alle möglichen Organe, gegen alle möglichen Krankheiten, über die zweckmässigsten Präparate, die Dosirung, die Gegengifte u. s.w. zu geben. Man kann sie gewissermassen nur mit Conversationslexici vergleichen, in denen man nach Bedarf einzelne Capitel nachschlägt, bei denen es aber Niemandem einfällt, sie von Anfang bis zun Ende durchzulesen.

Dies ist vielleiclit einer der Griinde, warum die Pharmakologie noch nicht so in's Blut und in den Saft der Aerzte übergegangen ist, weil sie ihnen nie in einer gesclimackhaften und zugleich leicht assinilirbaren Form geboten wurde, und jene daher nie dazu kamen, sich ein schönes klares Gesammtbild derselben zu machen.

Das Binz'sche Buch nun giebt die ganze Arzneimittellelıre in einer Reihe einzelner Ablandlungen in einer so schönen, anmuthenden Sprache, gewürzt mit so vielen interessanten historischen Rückblicken, in so klarer Darlegung des dem Arzte ans der ganzen Lehre Wissenswerthen, bei Hinweglassung aller zunächst unnöthig scheinenden Dinge, dass ich den mir so gut bekannten Stoff in solcher Form mit dem grössten Interesse und wirklicher Freude nochmals durchgelesen habe und davon wirklich gefesselt wurde. Man kann das Buch nicht besser charakterisiren, als indem man es als eine Sammlung geistvoller Feuilletons bezeichnet, die kein Arzt aus der Hand legen wird, ohne durch sie mit Interesse für diese so sclıwierige und sagen wir geradezu spröde Materie erfiillt worden zll sein. Insofern wird sie aber neben dem Angenehmen auch den grossen Nutzen bringen, dass die Aerzte, durch deren Lectüre eimmal für diese Materie interessirt, sich derselben in Zukunft mit viel grösserer Wärme annehmen, dass sie allmählich auch ihr therapeutisches Denken auf Grund viel reichhaltigeren pharmakologischen Wissens vertiefen und verbreitern und damit neben der Diagnostik auch die rationelle Therapie immer mehr zn Ehren kommen lassen. Es war höchsste Zeit, dass ein solches Buch kan, und wir frenen uns, dass es gekommen ist, dass es aus der Feder eines Mannes gekommen ist, der gerade wegen seiner vollständigen Beherrschung der enorm angeschwollenen Materie, wegen der langjälırigen Durcharbeitung nund Durchdringung derselben nach allen Richtungen am besten befäligt war, das Wichtige vom Unwichtigen zu scheiden und das Wichtige in so schöner, allgemein verstäıdlicher Sprache wiederzngeben d. h. im edelsten Sinne des Wortes zu popnlarisiren

Nach einer reizend geschriebenen Einleitming über die Begriffe und die Geschichte seiner Wissenschaft beginnt der Verfasser mit den beranschenden und betäubenden Mitteln, immer die besonders wichtigen und am meisten gebrauchten, wie Aether, Chloroform, Stickstoffoxydul, Chlorallydrat, Morphin n. s. w. in den Vordergrund stellend und zu 8 schönen Vorträgen abrundend, geht sodann zu den wichtigsten Alkaloiden, zum Jod und den ätherischen Oelen über, die er in 20 Vorträgen abhandelt, von da $\mathrm{zu}$ den Alkalien, Metallen und Metalloiden (in 10 Ablandlungen), sodaun zu den Antisepticis über und schliesst mit den Säuren, ausleerenden Mitteln, Fetten, Oelen Hautreizen und Aetzmittelı.

Alle diese Abliandlnngen stelien auf dem neuesten Standpunkte der Wissenschaft; es ist keine wichtige Arbeit anch der allerjüngsten Zeit iibersehen. Aber ebenso finden wir auch alle wichtigen Phasen, welche die Arzneimittel in der älteren Zeit erlebt haben, die Entdeckung, die Proben, die Irrtliïmer in kurzen, prägnanten Zügen geschildert, durch kleine Erzälllungen illustrirt, so dass, was die moderne Zeit wegen der Ueberfülle des auf sie eindringenden Stoffes immer melr vernachlässigen muss, anch die Pietät gegen die Pioniere der Wissenschaft nicht ausser Acht gelassen ist. Ich möchte gerade diese kleinen geschichtlichen Ausflüge, die der Verfasser fast bei jedem Arzneimittel unternimmt, fïr den ganz besonderen Reiz, den dieses Buch anf jeden Leser ausüben muss, mit verantwortlich machen.

Dabei ist auch der experimentelle Theil in den Abhandlungen ganz allgemeinverständlich gesclırieben; es schmücken das Buch vorzügliche Abbildungen; und anch die Ausstattung ist eine ganz vortreffliclie.

Wenn ich dalier dieses Buch ans voller Ueberzeugung zu recht fleissigem Studium empfehle, so bin ich mir bewusst, dass ich mir mit dieser Empfehlung den ganz besonderen Dank der Leser verdienen werde.

X. Billrotlı und Lücke. Handbucl der Frauenkrankheiten; Bd. II und III, zweite gänzlich umgearbeitete Anflage. Stuttgart; Ferd. Enke; 1886.

Der II. Band umfasst die Nenbildungen des Uterus von Prof. Dr. Gusserow; die Krankheiten der Ovarien von Prof. Dr. 0ls- 
hausen; die Krankheiten der Tuben, der Ligamente, des Beckenperitoneum und des Beckenbindegewebes, einschliesslich der Extrauterinschwangerschaft von Prof. Dr. Bandl.

Der III. Band umfasst die Krankheiten der weiblichen Brustdrüsen von Prof. Dr. Billroth; die Krankheiten der äusseren weiblichen Genitalien und der Dammrisse von Prof. Dr. Zweifel; die Krankheiten der weiblichen Harnröhre und Blase von Prof. Dr. Wiukel; die Krankheiten der Vagina von Prof. Dr. Breisky.

Band II enthält 113 in den Text gedruckte Holzschnitte, Band III deren 190.

Diesem Sammelwerke, welches das umfangreiche Material so erschöpfend und mustergültig nach Gehalt und Ausstattung zusammenfasst, ist kein zweites an die Seite zu stellen, und ist ihm die beste Aufnahme Seitens der Aerzte und Fachmänner sicher.

XI. F. v. Heuss. Chirurgisch-pathologische Tafeln. Lieferung $1-4$. Berlin. W. J. Peiser.

Die ersten vier Lieferungen (14 Tafeln) dieses $1875-77$ erscliienenen und s. Z. mit vielem Beifall aufgenommenen Atlas, dessen Fortsetzung der hohen Kosten der Ausführung wegen leider unterbleiben musste, sind von der Buchhandlung von W. J. Peiser (Friedrichstrasse 103) übernommen und werden statt des ursprünglichen Preises von 78 Mark für 30 Mark abgegeben. 